\title{
SPH Modelling of Hydrodynamic Lubrication along Rough Surfaces
}

\author{
Marco Paggi ${ }^{1, *} \mathbb{C}$, Andrea Amicarelli ${ }^{2}\left(\mathbb{D}\right.$ and Pietro Lenarda ${ }^{1}$ \\ 1 IMT School for Advanced Studies Lucca, Piazza San Francesco, 19, 55100 Lucca, Italy; \\ pietro.lenarda@imtlucca.it \\ 2 Ricerca sul Sistema Energetico-RSE SpA, Department SFE, via Rubattino, 54, 20134 Milan, Italy; \\ andrea.amicarelli@rse-web.it \\ * Correspondence: marco.paggi@imtlucca.it
}

Received: 1 August 2019; Accepted: 14 November 2019; Published: 21 November 2019

\begin{abstract}
Rough and textured surfaces are of paramount importance for lubrication, both in nature and in technology. While surface roughness relevantly influences both friction and wear, artificial surface texturing improves the performance of slider bearings as an energy efficiency action. The simulation of hydrodynamic lubrication by taking into account complex surfaces as boundaries requires the use of computational fluid dynamics (CFD) software able to predict the pressure and the velocity profile through the thickness of the fluid and at any point within the 3D domain. In the present study, a CFD-smoothed particle hydrodynamics (SPH) code is applied to simulate hydrodynamic lubrication for a linear slider bearing in the presence of a 3D rough surface, showing the capabilities of CFD-SPH in modelling such complex interaction phenomena. Numerical assessments involve the load capacity, the 3D fields of the velocity vector, and the pressure 3D field (both within the fluid domain and at the fluid-plate interface).
\end{abstract}

Keywords: SPH; bearings; fluid-structure interactions; roughness; linear sliders; hydrodynamic lubrication

\section{Introduction}

Hydrodynamic lubrication is the lubrication regime featured by a fluid film continuously interposed between interacting rigid solid surfaces and it represents a simplification of the elasto-hydrodynamic lubrication, in case deformation of the surface (elasticity effects) is negligible. Slider bearings are typically practical examples involving hydrodynamic lubrication. Slider bearings are widely employed in electric machines, turbomachinery, internal combustion engines, electric vehicles, hydraulic systems, medicine, automation, etc. Hereafter follows a brief introduction to numerical modelling of bearings for hydrodynamic lubrication.

To predict the response of lubricants in the hydrodynamic lubrication regime, a rigorous treatment of the fluid motion should consider the solution of the full Navier-Stokes equations, in which inertia, body, pressure and viscous terms are included [1]. However, there is a class of flow condition known as slow viscous motion in which the pressure and viscous terms prevail over the others, which leads to the Reynolds equations for hydrodynamic lubrication. Moreover, further simplification of Reynolds equations, to make them analytically solvable, is often put forward, by assuming only sliding motion and fluid properties constant in space, in addition to avoiding side leakage. All of this, which sometimes can be even further simplified by looking at the stationary regime, leads to a simple 1D differential equation for the pressure field. In [2], it has also been demonstrated that it is possible to derive the set of reduced equations for the classical lubrication approximation governing incompressible and iso-viscous flows from the full Navier-Stokes equations specialized for flows in thin gaps and applying dimensional analysis considerations. 
Based on the aforementioned theoretical framework, Williams and Symmons [3] developed a 1D computational fluid dynamics (CFD)-finite difference (FD) model to numerically reproduce the pressure longitudinal profiles within the fluid film of a linear slider. Dobrica and Fillon [4] developed a 2D CFD-finite volume method (FVM) code, alternatively using Navier-Stokes equations and Reynolds' equation for fluid films, and they validated it on the Rayleigh step bearings. They highlighted the importance of modelling the inertia terms, neglected by Reynolds' equation for fluid films. For step bearings, Vakilian et al. [5] found that neglecting the inertia terms in the momentum equations is responsible for underestimations at the leading edge and over-predictions at the trailing edge on the pressure field.

Almqvist et al. [6] provided inter-comparisons between a FD model based on Reynolds' equation for fluid films and a commercial CFD-FVM code based on Navier-Stokes equations. Almqvist et al. [6,7] also provided report analytical solutions on pressure longitudinal profiles, velocity vertical profiles, friction force, and load-bearing capacity (the frictional coefficient is the ratio between these two forces) for both a linear slider and the Rayleigh step slider, with null Dirichlet's boundary conditions for pressure. They also derived the optimal geometric configuration for a linear slider to maximize the load capacity (e.g., [3]), also referred to as load-bearing capacity or load-carrying capacity. Further, they analysed the effects of the surface roughness by means of the homogenization technique [8].

Rahmani et al. [9] presented an analytical approach based on Reynolds' equation for asymmetric partially textured slider bearings with surface discontinuities, to optimize the choice of the textures parameters with respect to the load capacity and the friction force.

Papadopoulos et al. [10] used a 2D CFD-FVM code to optimize micro-thrust bearings with surface texturing by means of numerical inter-comparisons. Fouflias et al. [11] used a commercial CFD-FVM code to simulate bearings with pockets/dimples and surface texturing, providing model inter-comparisons on steady-loads for different designs.

Paggi and Ciavarella [12] carefully investigated the role of roughness in contact mechanics, and Paggi and He [13] analysed the evolution of the free volume trapped between rough surfaces in contact, an essential parameter for the transition from hydrodynamic lubrication and mixed-lubrication regimes.

Gropper et al. [14] discussed a detailed review on hydrodynamic lubrication of textured surfaces, including (multi-scale) roughness effects and cavitation. Hajishaflee et al. [15] adopted a 2D CFD-FVM model to reproduce elasto-hydrodynamic lubrication problems for rolling element bearings, including cavitation effects. Snyder and Braun [16] proposed a perturbed Reynolds equation (PRE)-FD model. This is based on the representation of perturbed quantities (film thickness and pressure) within Reynolds' equation and provides three separated differential equations for static pressure, dynamic pressure associated with stiffness and dynamic pressure associated with damping.

Henry et al. [17] reported an experimental analysis for the start-up of thrust bearings under a transient regime. Mixed lubrication occurs before the stationary regime, which is, instead, characterized by hydrodynamic lubrication. This study highlights the importance of the texture geometry features in the formation of the fluid films and the reduction of the transient time to reach the lubrication regime, which minimizes the damage (stress concentrations over surface roughness peaks can lead to wear debris which deteriorate the bearing performance).

Pusterhofer et al. [18] presented a CFD-FVM model for modelling surface effects under hydrodynamic lubrication. On the use of Reynolds models for fluid films, they stated: "for structured surfaces the fluid flow cannot be represented correctly, due to the assumptions made when deriving the Reynolds equation" [18]. Their model was based on Navier-Stokes equations, was applied to a $3 \mathrm{D}$ rough lubrication gap at the micro-scale, and was coupled with a Reynolds-based model working at a coarser scale. They noticed that Navier-Stokes equations allows for the simulation of surface induced effects and that, "used in the classical sense, the Reynolds equation takes into account only the macroscopic geometry ... hydrodynamic effects because of the microscopic surface structure are not taken into account" [18]. Furthermore, the authors reported that "when the surface profile shows strong height changes, which can cause velocity changes in height direction ... the Reynolds equation 
is no longer valid to describe the flow through the rough gap" [18]. The authors highlighted the advantage of Navier-Stokes equations in providing greater detail of the fluid dynamics fields with respect to Reynolds models for fluid films, thus enabling to assess the effects of structured and textured surfaces under hydrodynamic lubrication.

Wang et al. [19] presented a parametric model for the optimization of groove texture profiles to improve the hydrodynamic lubrication performance of thrust bearings in terms of load capacity, friction coefficient and temperature rise.

Yildiran et al. [20] presented a 2D CFD-boundary element method (BEM) model for hydrodynamic lubrication, also including the representation of re-entrant textures. They highlighted the effects of roughness in inducing the so-called Stokes microscopic regime of lubrication. They also stated that in the "Stokes regime employing Stokes equations is essentially required" [20]. Stokes equations are a simplification of Navier-Stokes equations. At the same time, Stokes equations represent a generalization of Reynolds equations for fluid films because they permit a full 3D non-homogeneous and non-isotropic representation of the stress tensor gradients (and the volume forces).

Fernandez del Rincon et al. [21] proposed a gear transmission model to simulate the stress tensor field and the meshing forces under the hydrodynamic lubrication regime at low torques. The role played by the lubricant is also relevant in optimizing the gear acoustic performance (to minimize noise and provide an overall reduction of the noise vibration harshness effect).

Schvarts [22] analysed the effect of the microscopic roughness at the macro-scale under the mixed lubrication regime, where the solid surfaces are partially separated by the fluid, partially in direct contact by means of their asperities.

Recent generalizations and improvements of Reynolds' fluid film models concerning the indirect integration of additional features (e.g., surface roughness, slip conditions, non-Newtonian rheology, turbulence) have been devoted to overcome some of their intrinsic simplified model assumptions. On the other hand, the CFD codes are not limited by the above shortcomings and also permit the simulation of more complex features (e.g., transport of solid elements, particles and pollutants; erosion of the solid components; computation of the dynamics of the solid components depending on the fluid dynamics fields such as 2-way coupling), but are more complex and computationally demanding.

With respect to the state-of-the-art on CFD modelling for bearings, mostly based on 2D codes or Reynolds' simplified equation, the present study uses a 3D CFD code with all the terms of the Navier-Stokes equations for incompressible fluids with uniform viscosity. It also provides validations on local quantities (pressure and velocity profiles) and it is able to simulate complex 3D surfaces. The reference code for this study is the Free/Libre and Open Source Software (FOSS) CFD-smoothed particle hydrodynamics (SPH) code SPHERA (RSE SpA) [23], which assesses the load-bearing capacity, the 3D fields of the velocity vector $\underline{u}=(u, v, w)$, and the pressure 3D field both within the fluid domain and at the fluid-solid interfaces of a linear slider with a complex 3D surface introduced as boundary.

SPH is a mesh-less CFD method, whose computational nodes are represented by numerical fluid particles. Among the various numerical methods, SPH has several advantages: a direct estimation of free surface and phase/fluid interfaces; effective simulations of multiple moving bodies and particulate matter within fluid flows; direct estimation of Lagrangian derivatives (absence of non-linear advective terms in the balance equations); effective numerical simulations of fast transient phenomena; no meshing; simple non-iterative algorithms (in case the "weakly compressible" approach is adopted). On the other hand, SPH models are affected by the following drawbacks, if compared with mesh-based CFD tools: computational costs are slightly higher due to a larger stencil (around each computational particle), which causes a high number of interacting elements (neighbouring particles) at a fixed time step (nonetheless SPH codes are more suitable for parallelization); local refining of spatial resolution represents a current issue and is only addressed by few, advanced and complex SPH algorithms; accuracy is relatively low for classical CFD applications where mesh-based methods are well established (e.g., confined mono-phase flows). Detailed reviews on SPH assets and drawbacks are reported in [24]. Nevertheless, SPH models are effective in several, but peculiar, application fields. Some 
of them are here briefly recalled: flood propagation [25,26]; sloshing tanks [27]; gravitational surface waves [28]; hydraulic turbines [29]; liquid jets [29]; astrophysics and magneto-hydrodynamics [30]; body dynamics in free surface flows [31]; multi-phase and multi-fluid flows; sediment removal from water reservoirs [32]; and landslides [33,34].

In this article, the SPH formulation, whose mathematical and numerical formulations have been thoroughly described in [35] and validated in relation to benchmark fundamental solutions for hydrodynamic lubrication (uniform slider and a linear slider over flat-surface configurations), is applied to the simulation of the hydrodynamic lubrication regime for a linear slider bearing on a 3D microscopically rough surface. The effect of surface roughness in lubrication has been carefully investigated in the boundary lubrication regime, where surfaces are in contact and the friction coefficient is very high. However, even in the full-film hydrodynamic lubrication regime, friction may still be influenced and reduced by tailoring surface topographies. Even though there is no direct contact, the lubricant pressure may lead to stress concentrations high enough to cause fatigue, leading to excessive wear in the form of spalling in highly loaded situations [2]. In such cases, roughness can also affect the friction coefficient and, therefore, it is worth investigating its effect. Moreover, the simulation of full-film lubrication over textures arising from natural surfaces can also lead to important discoveries useful to design bio-inspired bearing solutions to further reduce friction and related energy losses.

\section{Linear Slider Bearing on a 3D Natural Rough Surface}

A linear slider is a system of two flat plates under relative motion and a fluid film between them. The slider is linear because the average film depth $h(\mathrm{~m})$-i.e., the fluid depth after filtering the micro-scale roughness - varies linearly with the distance from the plate edges. Herein the upper plate is mobile, whereas the lower plate is still and it is given by a complex (non-flat) 3D rough surface. The upper plate is featured by a leading edge (i.e., its most upstream face) and a trailing edge (i.e., its most downstream face). The slope angle between the mobile plate of the particular bearing of this study and the horizontal is $\alpha=0.568^{\circ}$. This value is relevant as $h<<L$ (a hypothesis of Reynolds' equation for fluid films), where $L(\mathrm{~m})$ is the bearing length.

To demonstrate the applicability of SPH in simulating hydrodynamic lubrication with complex rough boundaries, a strip of a tomato leaf acquired using the confocal profilometer Leica DCM3D available in the MUSAM Lab of the Multiscale Analysis of Materials (MUSAM) research unit of the IMT School for Advanced Studies Lucca has been used as the lower boundary, see also the open data project Wiki Surface [36]. This rigid tomato leaf is representative of a textured surface with geometrical complexity and roughness scale comparable with the artificial textures used to optimize linear sliders. Grid interpolator software [37] has been used to elaborate the original tomato leaf surface as a grid-based data interpolator with modifications on the spatial resolution and the despiking procedure, as described in the following Figures 1-3.

Values greater than a positive threshold or smaller than a negative threshold have been discarded, being considered out-layers affected by profilometer measurement errors. The thresholds are provided as input data in terms of normalized variables. The upper threshold is $t_{u}=m_{z}+n t_{u} \cdot \sigma_{z}$, where $m_{z}(\mathrm{~m})$ is the average height, $\sigma_{z}=1.81 \times 10^{-5} \mathrm{~m}$ the height standard deviation and $n t_{u}=3.5$ a non-dimensional input factor. Analogously, the lower threshold is $t_{l}=m_{z}-n t_{u} \cdot \sigma_{z}$, with $n t_{l}=3.5$. The interpolation influence radius provided as an input normalized influence radius $\left(r_{i n}=10\right)$. A Shepard interpolation has been carried out, with the distance exponent provided as an input datum $\left(e_{d}=6\right)$. The despiking procedure described above allows the outliers of the raw surface to be filtered (Figures 2 and 3). 


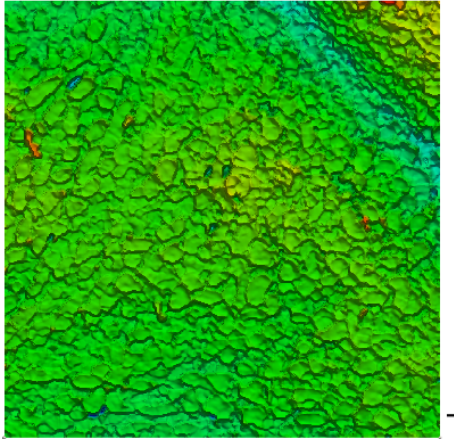

(a)

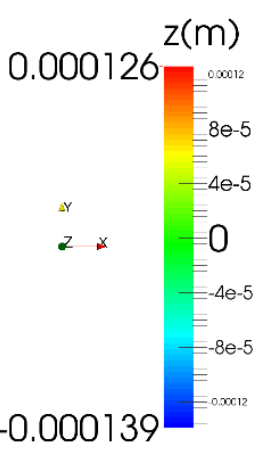

0.000139
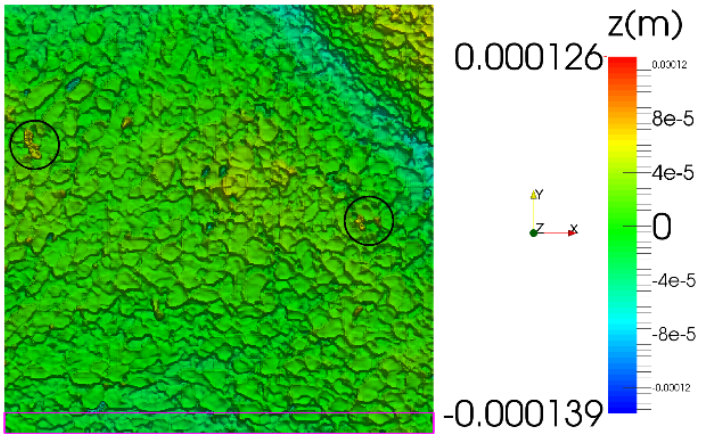

(b)

Figure 1. Rigid tomato leaf 3D surface. (a): surface elaborated without despiking. (b): surface elaborated with despiking. Top view. The violet rectangle on the bottom of the right panel denotes the strip used for the smoothed particle hydrodynamics (SPH) simulation (this strip is featured by the lowest trend among the leaf strips thus minimizing the maximum film thickness between the bearing plates). The black circumferences on the right panel delimit two examples of regions where despiking effects are more relevant.

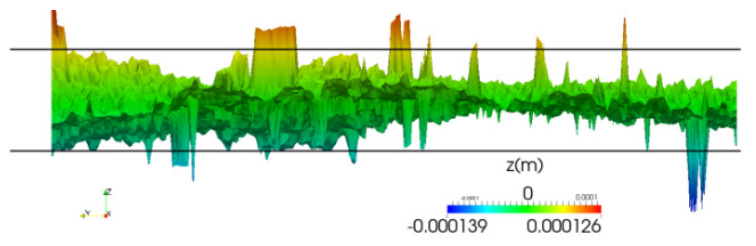

(a)

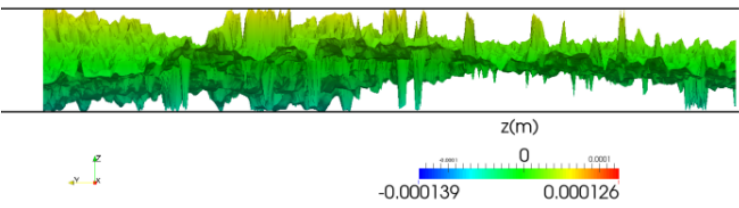

(b)

Figure 2. Rigid tomato leaf 3D surface. (a): surface elaborated without despiking. (b): surface after despiking. Lateral view.

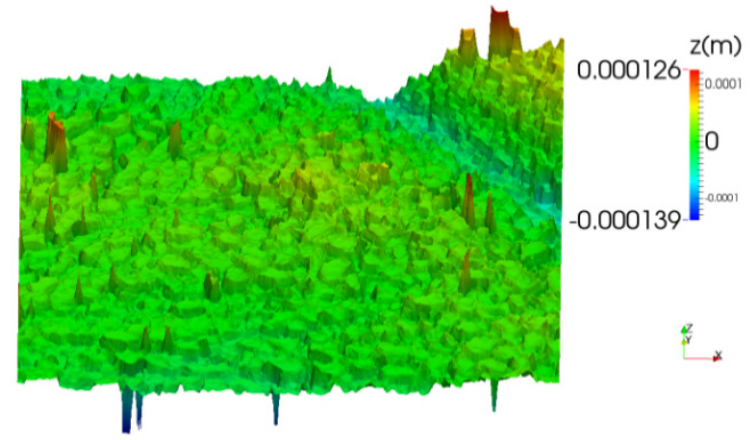

(a)

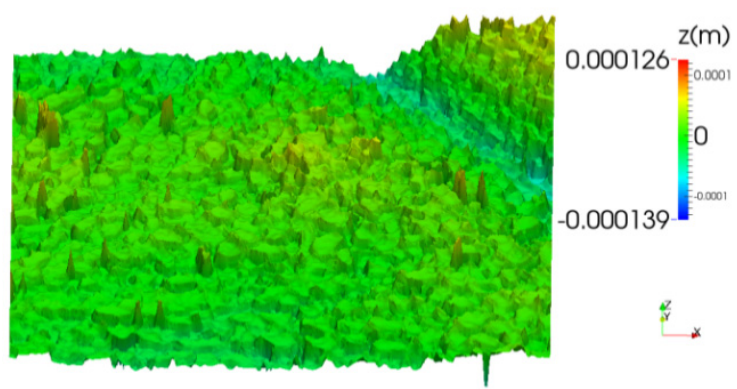

(b)

Figure 3. Rigid tomato leaf 3D surface. (a): surface elaborated without despiking. (b): surface after despiking. 3D view.

For the CFD simulation, the initial fluid velocity $u_{f, 0}(\mathrm{~m} / \mathrm{s})$ has been set as the average velocity of the solid plates. As the lower plate is still, then $u_{f, 0}=u_{s, 1} / 2$, where " $s, 1$ " denotes the upper plate. The reference velocity $U^{*}=u_{s, 1}=50 \mathrm{~m} / \mathrm{s}$ determines a Reynolds number $(R e=c a$. 100) which guarantees a laminar regime within most of the bearing film (far enough from the mobile plate edges and the roughness elements). Omitting gravity is equivalent and alternative to replacing pressure with the reduced pressure (i.e., the difference between pressure and its hydrostatic component). The domain is laterally confined by vertical solid walls, where a boundary treatment scheme is applied [23].

The domain length and width selected for the simulation were $L_{d o m}=8.48 \times 10^{-4} \mathrm{~m}$ and $W_{\text {dom }}=4.2 \times 10^{-5} \mathrm{~m}$, respectively. The domain depth is $H_{\text {dom }}=9.5 \times 10^{-5} \mathrm{~m}$. The slider length was $L=4.24 \times 10^{-4} \mathrm{~m}$. The initial position of the plate barycentre was represented by the horizontal 
coordinates $x_{C M, 0}=0.35 \cdot L_{d o m}=2.968 \times 10^{-4} \mathrm{~m}, y_{\mathrm{CM}, 0}=W_{\text {dom }} / 2=2.1 \times 10^{-5} \mathrm{~m}$. The above length scales are chosen to impose that the sliding lasts enough to dynamically establish stationary conditions, to reproduce all the roughness elements of the fixed plate, the fluid film and the mobile plate, and to avoid useless computational demand. The upper plate inclination (with respect to the horizontal) is expressed by the slope angle $\alpha=9.91 \times 10^{-3} \mathrm{rad}\left(0.568^{\circ}\right)$. The dynamic viscosity of the fluid is $\mu=319 \times 10^{-3} \mathrm{~Pa} \cdot \mathrm{s}$, representative of the motor oil "SAE $40^{\prime \prime}$ at ambient conditions, being the oil density equal to $\rho=900 \mathrm{~kg} / \mathrm{m}^{3}$. This lubricant is still before the sliding of the mobile upper plate over the fixed plate of the bearing. The upstream and downstream fluid frontiers were modelled as open boundaries. The vertical and longitudinal monitoring lines for the profiles were located along the domain centreline $\left(y=W_{\text {dom }} / 2\right)$.

The spatial resolution for the present problem is defined by $d x=1.6 \times 10^{-6} \mathrm{~m}, h_{S P H} / d x=1.3$ and $d x / d x_{s}=2$, where $h_{S P H}$ is the SPH influence length, $d x$ is the spatial resolution, the subscripts " $f$ " and "s" represent the fluid and solid SPH particles, respectively.

Results are reported in terms of non-dimensional quantities for pressure $p(\mathrm{~Pa})$, velocity magnitude and time $t(\mathrm{~s})$ :

$$
p^{*} \equiv \frac{p h^{2}}{\mu U L}, U^{*} \equiv\left|u_{s, 1}\right|+\left|u_{s, 2}\right|, T \equiv \frac{2 t U^{*}}{h_{0}}
$$

where $p^{*}$ is defined as normalized pressure and $h_{0}(\mathrm{~m})$ is the representative oil depth (here equal to its minimum value), with $h_{0} / L=1 / 25 L$ represents the upper plate length and $h_{0}$ is the minimum non-null value of $h$-i.e., the mobile upper plate and the rough bottom plate are never in direct contact):

$$
\varepsilon \equiv \frac{h_{0}}{L}<<1 ; p \propto \varepsilon^{-2}, \varepsilon \rightarrow 0
$$

with the upper plate width equal to the domain width.

The following results refers to the final time step, which is equal to the physical time and representative of stationary conditions $\left(T=16.9\right.$, i.e., $\left.t=3.00 \times 10^{-6} \mathrm{~s}\right)$. Considering both the extremely small ratios of the involved length scales and the temporary unavailability of the best machine architecture usually adopted by the code, the simulation lasted $23.4 \mathrm{~h}$ on 68 cores Intel Xeon Phi7250 (Knight Landings, Intel, Santa Clara, CA, USA) at 1.4 GHz.

Figure 4 shows a lateral view of a representative 3D field of the $x$-component of the normalized velocity. No-slip conditions are well reproduced at both the fixed frontier and the plate bottom. Within the confined fluid area, the velocity horizontal gradient depends on the local oil depth and the proximity to the edges of the mobile plate.

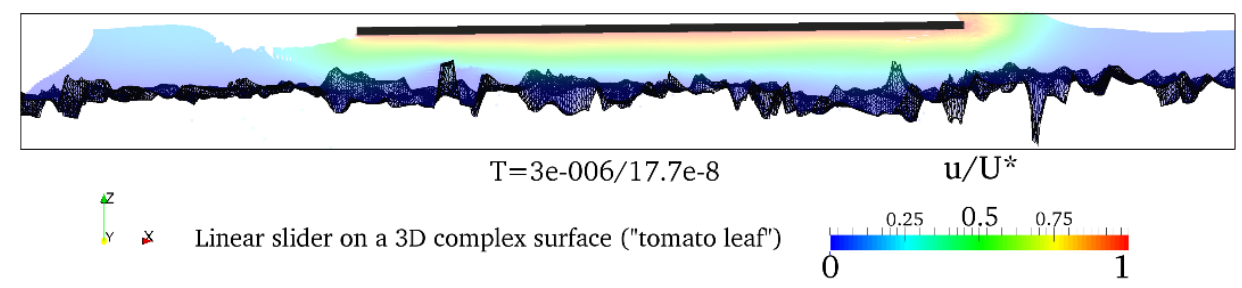

Figure 4. Linear slider on a processed rigid tomato leaf. Non-dimensional velocity 3D field (lateral view). $T$ is the non-dimensional time defined within the text. The upper plate moves towards right.

Figure 5 reports a representative lateral view of the 3D field of the normalized pressure. The leading edge and the trailing edge are locally featured by an over-pressure stagnation region and an under-pressure zone, respectively. The normalized pressure field is strongly perturbed by the asperities of the bottom complex surface. 


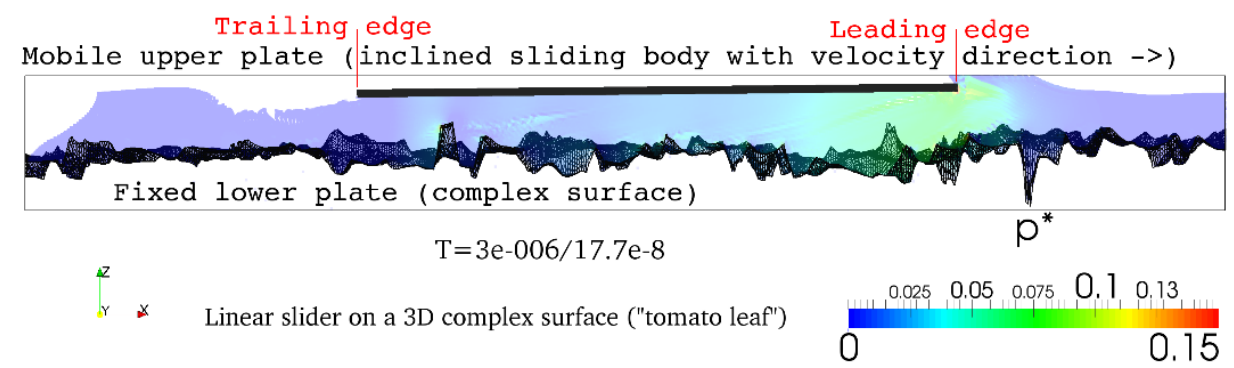

Figure 5. Linear slider on processed a rigid tomato leaf. 3D field of the normalized pressure within the fluid sub-domain (lateral view). $T$ is the non-dimensional time defined within the text. The upper plate moves towards right (higher $x$ values).

Figure 6 shows a representative 3D view of the field of the $x$-component of the normalized velocity. The 3D effects (dependence on the $y$-coordinate), due to the complex shape of the bottom surface, are relevant. The field of the $y$-component of the normalized velocity is not reported because it is negligible (with respect to the $x$-component) since the stationary regime is achieved. Figure 7 shows a representative 3D view of the field of the $z$-component of the normalized velocity. The absolute value of the biggest positive value is $50 \%$ of the velocity scale and occurs at the leading edge. The absolute value of the smallest negative value is $24 \%$ of the velocity scale and occurs at the trailing edge. Figure 8 reports a representative 3D view of the normalized velocity both in terms of vector field and scalar absolute value. This example image integrates the information coming from the three velocity components simulated all over the fluid domain and demonstrates the importance of simulating the vertical component of the velocity vector, especially near the leading and trailing edges of the mobile plate.

Figure 9 reports a representative 3D view of the normalized pressure field at the interface between the oil and the upper plate. The presence of a complex 3D bottom surface introduces local fluctuations of the pressure field along the $x$-axis as local perturbations to the parabolic shape of the pressure longitudinal profiles (Figure 11a): at the micro-scale the roughness elements represent obstacles featured by stagnation zones on the upstream faces and under-pressure regions on the downstream faces.

Comparing Figures 4-9, one notices that the 3D effects are mitigated with $z$. By contrast with the codes based on the simplified Reynolds' equation for fluid films, this SPH code can represent the time and space evolution of the velocity vector, not just the stationary regime for the $x$-component of velocity.

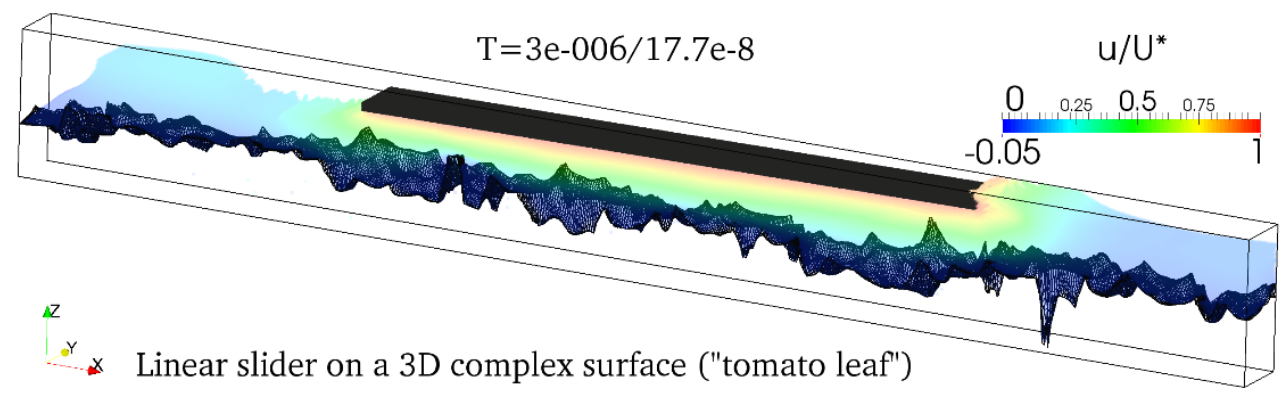

Figure 6. Linear slider on a processed rigid tomato leaf. 3D field of the $x$-component of the non-dimensional velocity (3D view). 


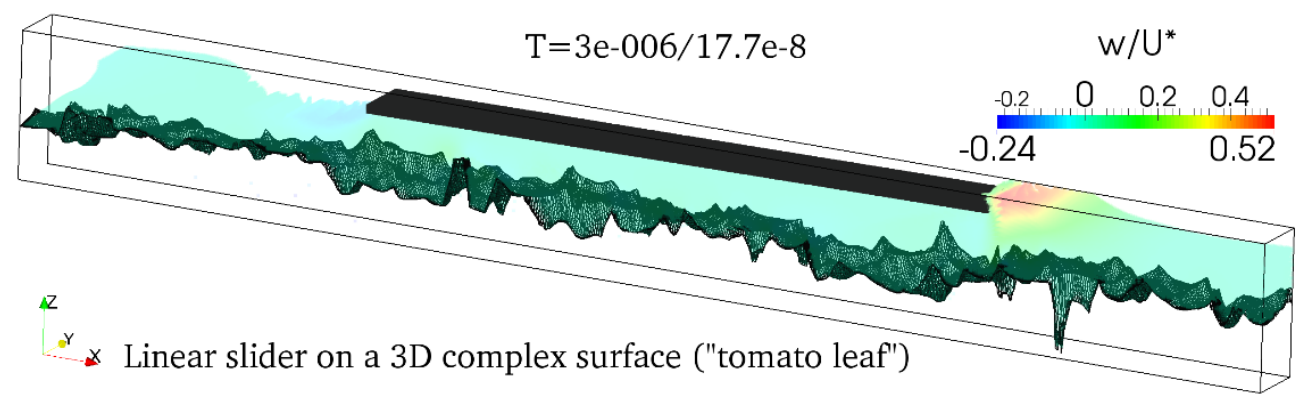

Figure 7. Linear slider on a processed rigid tomato leaf. 3D field of the $z$-component of the non-dimensional velocity (3D view).

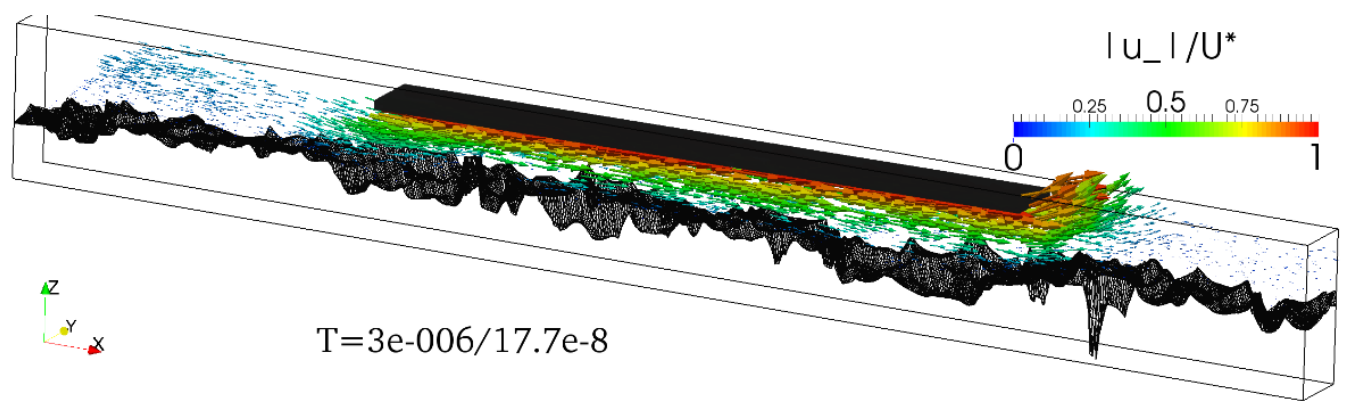

Figure 8. Linear slider on a processed rigid tomato leaf. 3D scalar (absolute value) and vector fields of the non-dimensional velocity (3D view).

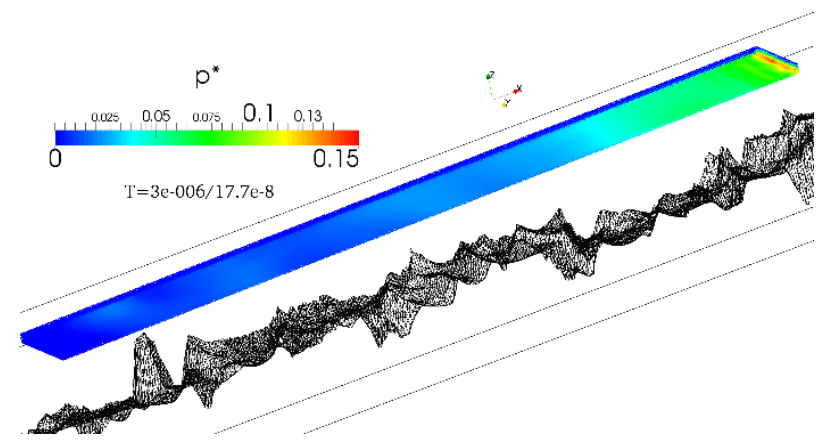

Figure 9. Linear slider on a processed rigid tomato leaf. 3D field of the normalized pressure within the solid sub-domain (bottom view). The view angle is different from the other 3D-view figures.

The simulation reference system is defined by the axis orientation of Figures 4 and 6 (with the origin located at the lower left corner in the plan view-i.e., non-null values are used for the horizontal coordinates $x$ and $y-)$. At the same time, in order to monitor the simulated profiles, a local reference system is assumed with non-dimensional spatial coordinates, as explained below.

Figure 10 shows the projection of the absolute value of the non-dimensional 3D velocity field on different planes parallel to the flow direction at different heights $z$. On these planes, the components of the $3 \mathrm{D}$ velocity vector field are characterized by a significant inhomogeneity along each one of the three directions aligned with the Cartesian axes. It is observed that as the $z$ coordinate increases, the velocity field along the corresponding plane is more affected by the velocity of the upper mobile plate.

Figure 11a reports the normalized pressure longitudinal profile, from the inlet section (leading edge, $X=1$ ) to the outlet section (trailing edge, $X=0$ ) of the slider. Within these sections, the 2D pressure field is not imposed, but dynamically simulated until stationary conditions are achieved. The pressure field at the reference plot refers to the surface body particles representing the fluid-body interface (inclined monitoring line). The other three plots are monitored within the fluid domain at different non-dimensional heights $Z=\left(z-z_{\text {bot }}\right) / h$ : $Z_{1}=1, Z_{2}=0.5, Z_{3}=0$ (horizontal monitoring lines; 
$z_{b o t}$ is the local height of the lower plate). Results are plotted only within the bearing region (i.e., the portion of the fluid film laying between the upper plate and the bottom). The higher the position of the monitoring line (far from the asperities of the complex surface) the smoother the normalized pressure profile is. The two upper profiles are affected by a more pronounced estimation in the normalized pressure maxima at the leading edge. These two profiles provide very close results: some discrepancies are visible around the leading edge where the relative distance of these monitoring lines is maximum. By contrast with the analytical solutions (e.g., [6]) and the codes based on the simplified Reynolds' equation for fluid films, this SPH code can simulate non-null vertical gradients of the pressure field (as in [38]), it takes into account the inertial effects due to the fluid-structure interactions at the leading and the trailing edges, and the profile concavity is directed upward.

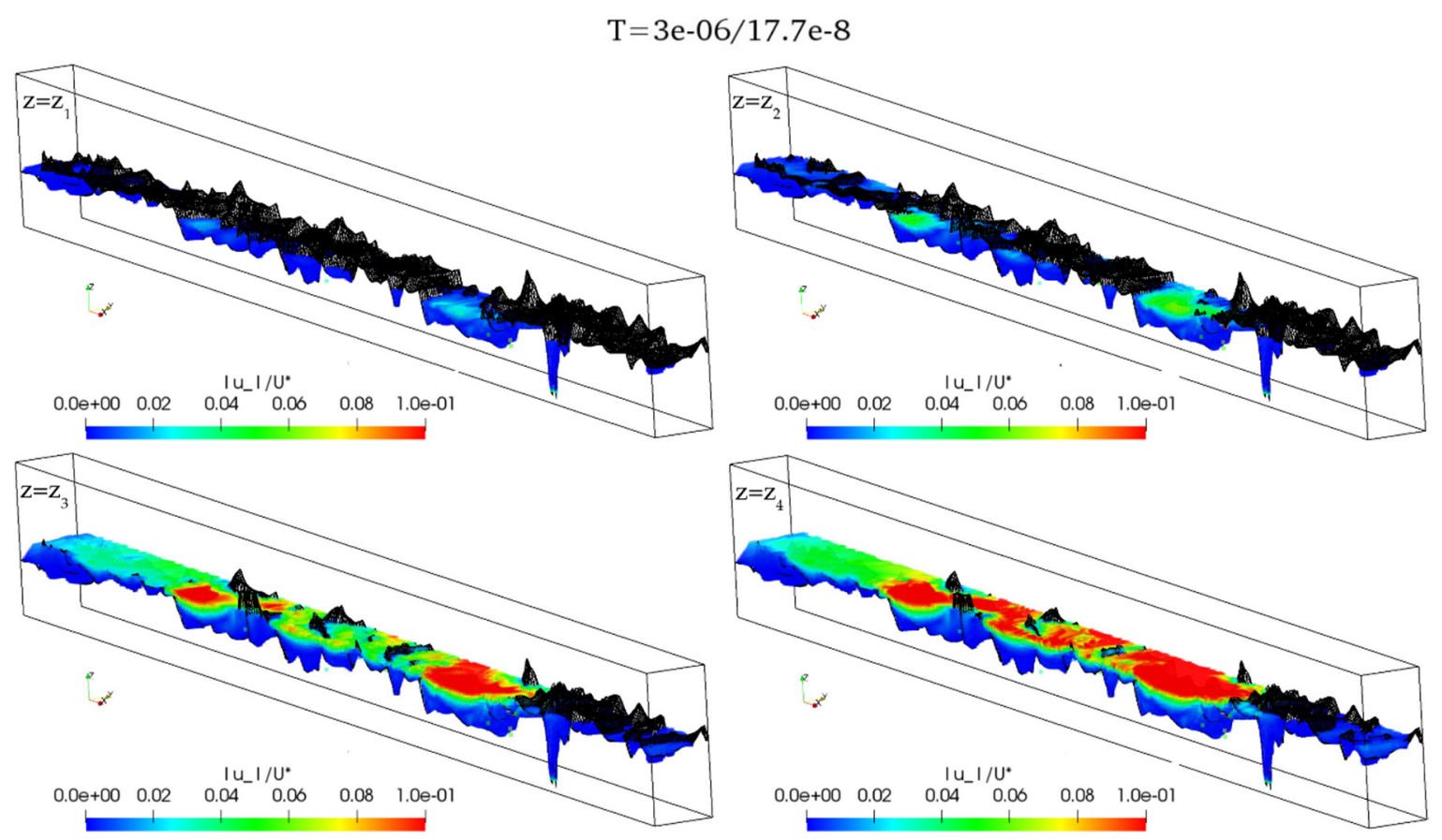

Figure 10. Linear slider on a processed rigid tomato leaf. 3D scalar field (absolute value) of the non dimensional velocity (3D view) at different heights $z=z_{i}, i=1, \ldots, 4$ where $z_{1}=-2.8 \times 10^{-5} \mathrm{~m}$, $z_{2}=-2.4 \times 10^{-5} \mathrm{~m}, z_{3}=-2.0 \times 10^{-5} \mathrm{~m}, z_{4}=-1.8 \times 10^{-5} \mathrm{~m}$. The view angle is different from the other 3D-view figures.

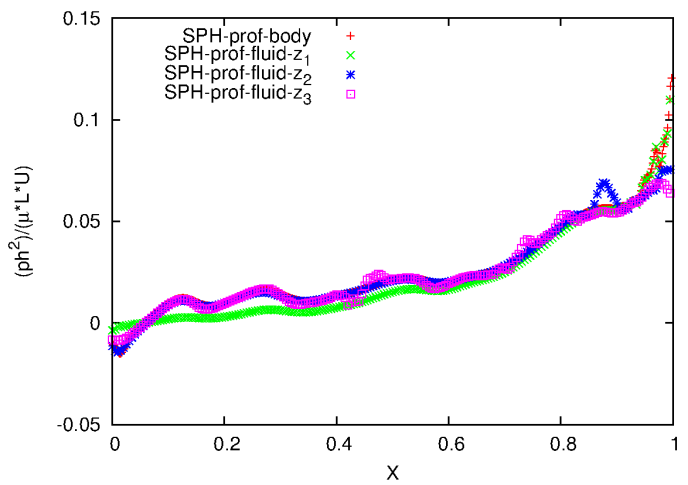

(a)

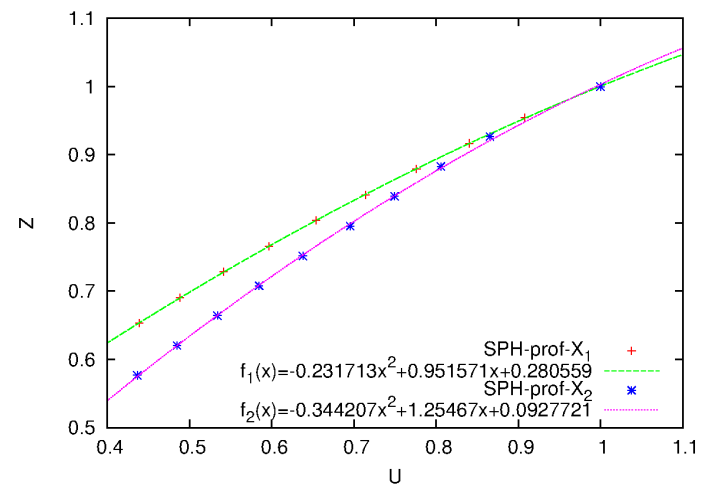

(b)

Figure 11. Linear slider on a processed rigid tomato leaf: normalized pressure longitudinal profiles (a) and velocity vertical profiles $(\mathbf{b})$. 
Figure $11 \mathrm{~b}$ shows two vertical profiles of the $x$-component of the normalized velocity. The numerical probes are located at $X_{1}=0.74$ and $X_{2}=0.98$, where $X$ is the non-dimensional distance from the trailing edge $\left(x-x_{0}\right) / L$ and $x_{0}(\mathrm{~m})$ is the $x$ coordinate of the trailing edge of the upper mobile plate at a fixed time. The concavity of the parabolic profile (directed downwards) increases as long as the monitoring position is located at a further distance from the leading edge. This feature is similar to what happens for a linear slider bearing over a flat bottom surface, where analytical solutions do apply. However, by contrast with the analytical solutions and the codes based on the simplified Reynolds' equation for fluid films, the concavity of the velocity profile close to the leading edge is directed downward. As for linear sliders over a flat bottom (and the results from Reynolds' equations for fluid films), the vertical profile of the horizontal velocity is quadratic in $z$ and it depends on $x$.

As the spatial discretization of the numerical pressure profile is uniform, the numerical non-dimensional load-bearing capacity $L_{C}$ is estimated as the average of the normalized pressure values over the mobile plate bottom (the squared parentheses represent an SPH estimation):

$$
\left\langle L_{C}\right\rangle \equiv \frac{\left\langle l_{c}\right\rangle}{\frac{\mu U L^{2}}{h^{2}}}=\frac{h^{2}}{\mu U L^{2}} \sum_{i=1, N} p_{s, i} d x_{i}=\frac{1}{N} \sum_{i=1, N} p_{s, i}^{*}
$$

where $N$ represents the number of surface body particles along the centreline of the mobile plate bottom. The estimated non-dimensional load-bearing capacity for the above simulation is $L_{C}=0.026$.

\section{Conclusions}

A computational fluid dynamics-smoothed particle hydrodynamics (CFD-SPH) code has been used to simulate a linear slider bearing in the presence of a 3D complex surface. Numerical assessments show the versatility and the robustness of the computational framework for the simulation of the fluid flow and the prediction of the load-bearing capacity, the complete 3D fields of the velocity vector, as well as the pressure 3D field (both within the fluid domain and at the fluid-plate interface). This study proves the capabilities of CFD-SPH modelling in representing hydrodynamic lubrication phenomena in the presence of 3D complex surfaces.

The following key physical features arise from the proposed analysis. The presence of a complex 3D bottom surface introduces local fluctuations of the pressure field along the motion direction of the bearing mobile plate. The 3D effects are mitigated with the distance from the complex surface. By contrast with the analytical solutions and the codes based on the simplified Reynolds' equation for fluid films, this SPH code predicts the time and space evolution of the velocity vector (not just the stationary regime for the $x$-component of velocity). The more the monitoring line is far from the asperities of the complex surface, the smoother the normalized pressure profile is. The normalized pressure grows with height at the leading edges. By contrast with the analytical solutions and the codes based on the simplified Reynolds' equation for fluid films, this SPH code can simulate non-null vertical gradients of the pressure field, takes into account the inertial effects due to the fluid-structure interactions at the leading and the trailing edges, and the pressure profile concavity is directed upward. The more the velocity profile is distant from the leading edge, the more the profile concavity (directed downward) is pronounced. This feature is similar to linear slider bearings over flat bottom. However, contrarily to the analytical solutions and the codes based on the simplified Reynolds' equation for fluid films, the concavity of the velocity profile close to the leading edge is directed downward. As for linear sliders over the flat bottom (and the results from Reynolds' equations for fluid films), the vertical profile of the horizontal velocity is quadratic in $z$ and depends on $x$. The code herein used (SPHERA) is developed and distributed on a GitHub public repository. The successful application of SPHERA for hydrodynamic lubrication on complex surfaces opens new research perspectives that can be handled within a SPH computational fluid dynamics framework, such as, for instance, the prediction of the movement of particles dispersed in the fluid, as well as erosion of the rough boundary. Moreover, 
combined with a topology optimization framework, it allows the design of the topology of complex textured surfaces to enhance lubrication.

Author Contributions: The authors equally contributed to the CFD simulations and in writing the manuscript. The CFD code SPHERA has been updated to deal with rough boundaries by A.A., based on the scientific discussion with M.P.

Funding: This research received no external funding.

Acknowledgments: The authors acknowledge the CINECA award under the ISCRA initiative, for the availability of High Performance Computing resources and support. In particular, SPHERA simulations have been supported by means of the following instrumental funding HPC projects: HSPHCS9; HSPHER9b. MP would like to thank Claudia Borri for contributing to surface roughness measurements in the MUSAM-Lab. The contribution of the author affiliated at RSE SpA has been financed by the Research Fund for the Italian Electrical System (for "Ricerca di Sistema -RdS-"), in compliance with the Decree of Minister of Economic Development 16 April 2018. SPHERA v.9.0.0 is realised by RSE SpA thanks to the funding "Fondo di Ricerca per il Sistema Elettrico" within the frame of a Program Agreement between RSE SpA and the Italian Ministry of Economic Development (Ministero dello Sviluppo Economico).

Conflicts of Interest: The authors declare no conflict of interest.

\section{References}

1. Hamrock, B.J.; Schmid, S.R.; Jacobson, B.O. Fundamentals of Fluid Film Lubrication, 2nd ed.; CRC Press: Boca Raton, FL, USA, 2004.

2. Almqvist, A.; Pérez-Ràfols, F. Modelling Flows in Lubrication. In Modeling and Simulation of Tribological Problems in Technology; Paggi, M., Hills, D., Eds.; CISM International Centre for Mechanical Sciences, Courses and Lectures; Springer Nature Switzerland AG: Cham, Switzerland, 2020; Volume 593, Chapter 6.

3. Williams, P.D.; Symmons, G.R. Analysis of hydrodynamic slider thrust bearings lubricated with non-Newtonian fluids. Wear 1987, 117, 91-102. [CrossRef]

4. Dobrica, M.; Fillon, M. Reynolds' Model Suitability in Simulating Rayleigh Step Bearing Thermohydrodynamic Problems. Tribol. Trans. 2005, 48, 522-530. [CrossRef]

5. Vakilian, M.; Gandjalikhan Nassab, S.A.; Kheirandish, Z. CFD-Based Thermohydrodynamic Analysis of Rayleigh Step Bearings Considering an Inertia Effect. Tribol. Trans. 2014, 57, 123-133. [CrossRef]

6. Almqvist, A. On the Effects of Surface Roughness in Lubrication. Ph.D. Thesis, Lulea University of Technology, Lulea, Sweden, 2006.

7. Almqvist, T.; Almqvist, A.; Larsson, R. A comparison between computational fluid dynamic and Reynolds approaches for simulating transient EHL line contacts. Tribol. Int. 2004, 37, 61-69. [CrossRef]

8. Almqvist, A.; Essel, E.K.; Persson, L.E.; Wall, P. Homogenization of the unstationary incompressible Reynolds equation. Tribol. Int. 2007, 40, 1344-1350. [CrossRef]

9. Rahmani, R.; Mirzaee, I.; Shirvani, A.; Shirvani, H. An analytical approach for analysis and optimisation of slider bearings with infinite width parallel textures. Tribol. Int. 2010, 43, 1551-1565. [CrossRef]

10. Papadopoulos, C.I.; Nikolakopoulos, P.G.; Kaiktsis, L. Evolutionary Optimization of Micro-Thrust Bearings with Periodic Partial Trapezoidal Surface Texturing. J. Eng. Gas Turbines Power 2011, 133, 1-10. [CrossRef]

11. Fouflias, D.G.; Charitopoulos, A.G.; Papadopoulos, C.I.; Kaiktsis, L.; Fillon, M. Performance comparison between textured, pocket, and tapered-land sector-pad thrust bearings using computational fluid dynamics thermohydrodynamic analysis. J. Eng. Tribol. 2015, 229, 376-397. [CrossRef]

12. Paggi, M.; Ciavarella, M. The coefficient of proportionality $\kappa$ between real contact area and load, with new asperity models. Wear 2010, 268, 1020-1029. [CrossRef]

13. Paggi, M.; He, Q.C. Evolution of the free volume between rough surfaces in contact. Wear 2015, 336, 86-95. [CrossRef]

14. Gropper, D.; Wang, L.; Harvey, T.J. Hydrodynamic lubrication of textured surfaces: a review of modeling techniques and key findings. Tribol. Int. 2016, 94, 509-529. [CrossRef]

15. Hajishafiee, A.; Kadiric, A.; Ioannides, S.; Dini, D. A coupled finite-volume CFD solver for two-dimensional elastohydrodynamic lubrication problems with particular application to rolling element bearings. Tribol. Int. 2017, 109, 258-273. [CrossRef]

16. Snyder, T.; Braun, M. Comparison of Perturbed Reynolds Equation and CFD Models for the Prediction of Dynamic Coefficients of Sliding Bearings. Lubricants 2018, 6. [CrossRef] 
17. Henry, Y.; Bouyer, J.; Fillon, M. Experimental analysis of the hydrodynamic effect during start-up of fixed geometry thrust bearings. Tribol. Int. 2018, 120, 299-308. [CrossRef]

18. Pusterhofer, M.; Bergmann, P.; Summer, F.; Grün, F.; Brand, C. A Novel Approach for Modeling Surface Effects in Hydrodynamic Lubrication. Lubricants 2018, 6. [CrossRef]

19. Wang, W.; He, Y.; Zhao, J.; Mao, J.; Hu, Y.; Luo, J. Optimization of groove texture profile to improve hydrodynamic lubrication performance: Theory and experiments. Friction 2018. [CrossRef]

20. Yıldıran, İ.N.; Temizer, I.; Cetin, B. Homogenization in Hydrodynamic Lubrication: Microscopic Regimes and Re-Entrant Textures. J. Tribol. 2018, 140, 011701. [CrossRef]

21. Fernandez-Del-Rincon, A.; Diez-Ibarbia, A.; Theodossiades, S. Gear transmission rattle: Assessment of meshing forces under hydrodynamic lubrication. Appl. Acoust. 2019, 144, 85-95. [CrossRef]

22. Shvarts, A. Couplage Mécano-Fluidique pour le Contact et le Frottement a Petites et à Grandes échelles. Ph.D. Thesis, Université PSL MINES Paris Tech, Paris, France, 2019.

23. SPHERA (RSE SpA). Available online: https://github.com/AndreaAmicarelliRSE/SPHERA (accessed on 18 November 2019).

24. Amicarelli, A.; Kocak, B.; Sibilla, S.; Grabe, J. A 3D Smoothed Particle Hydrodynamics model for erosional dam-break floods. Int. J. Comput. Fluid Dyn. 2017, 31, 413-434. [CrossRef]

25. Gu, S.; Zheng, X.; Ren, L.; Xie, H.; Huang, Y.; Wei, J.; Shao, S. SWE-SPHysics simulation of dam break flows at South-Gate Gorges Reservoir. Water 2017, 9, 387. [CrossRef]

26. Vacondio, R.; Rogers, B.D.; Stansby, P.K.; Mignosa, P. SPH Modeling of Shallow Flow with Open Boundaries for Practical Flood Simulation. J. Hydraul. Eng. 2011, 138, 530-541. [CrossRef]

27. Amicarelli, A.; Agate, G.; Guandalini, R. A 3D Fully Lagrangian Smoothed Particle Hydrodynamics model with both volume and surface discrete elements. Int. J. Numer. Methods Eng. 2013, 95, 419-450. [CrossRef]

28. Colagrossi, A.; Souto-Iglesias, A.; Antuono, M.; Marrone, S. Smoothed-particle-hydrodynamics modeling of dissipation mechanisms in gravity waves. Phys. Rev. E 2013, 87, 023302. [CrossRef] [PubMed]

29. Marongiu, J.C.; Leboeuf, F.; Caro, J.; Parkinson, E. Free surface flows simulations in Pelton turbines using an hybrid SPH-ALE method. J. Hydraul. Res. 2010, 48, 40-49. [CrossRef]

30. Price, D.J. Smoothed Particle Hydrodynamics and Magnetohydrodynamics. J. Comput. Phys. 2012, 231, 759-794. [CrossRef]

31. Amicarelli, A.; Albano, R.; Mirauda, D.; Agate, G.; Sole, A.; Guandalini, R. A Smoothed Particle Hydrodynamics model for 3D solid body transport in free surface flows. Comput. Fluids 2015, 116, 205-228. [CrossRef]

32. Manenti, S.; Sibilla, S.; Gallati, M.; Agate, G.; Guandalini, R. SPH Simulation of Sediment Flushing Induced by a Rapid Water Flow. J. Hydraul. Eng. ASCE 2011, 138, 227-311. [CrossRef]

33. Abdelrazek, A.M.; Kimura, I.; Shimizu, Y. Simulation of three-dimensional rapid free-surface granular flow past different types of obstructions using the SPH method. J. Glaciol. 2016, 62, 335-347. [CrossRef]

34. Bui, H.H.; Fukagawa, R.; Sako, K.; Ohno, S. Lagrangian meshfree particles method (SPH) for large deformation and failure flows of geomaterial using elastic-plastic soil constitutive model. Int. J. Numer. Anal. Meth. Geomech. 2008, 32, 1537-1570. [CrossRef]

35. Paggi, M.; Amicarelli, A.; Lenarda, P. SPH modelling of hydrodynamic lubrication: laminar fluid flow-structure interaction with no-slip conditions for slider bearings. arXiv 2019, arXiv:1910.04561, and submitted to Computational Particle Mechanics.

36. Wiki Surface. Available online: http://musam.imtlucca.it/wikisurf.html (accessed on 18 November 2019).

37. Grid Interpolator v.2.0 (RSE SpA). Available online: https://github.com/AndreaAmicarelliRSE/Grid_ Interpolator (accessed on 18 November 2019).

38. Shyu, S.H.; Hsu, W.C. A Numerical Study on the Negligibility of Cross-Film Pressure Variation in Infinitely Wide Plane Slider Bearing, Rayleigh Step Bearing and Micro-Grooved Parallel Slider Bearing. Int. J. Mech. Sci. 2018, 137, 315-323. [CrossRef]

(C) 2019 by the authors. Licensee MDPI, Basel, Switzerland. This article is an open access article distributed under the terms and conditions of the Creative Commons Attribution (CC BY) license (http://creativecommons.org/licenses/by/4.0/). 